\title{
Nanoscale
}

PAPER
View Article Online

View Journal | View Issue
Check for updates

Cite this: Nanoscale, 2017, 9, 14405

\section{Hybrid magnetic iron oxide nanoparticles with tunable field-directed self-assembly $\dagger$}

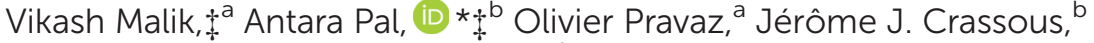 \\ Simon Granville, ${ }^{c}$ Bernard Grobety, ${ }^{d}$ Ann M. Hirt, ${ }^{e}$ Hervé Dietsch $\S^{a}$ and \\ Peter Schurtenberger (iD) $* b$
}

We describe the synthesis of hybrid magnetic ellipsoidal nanoparticles that consist of a mixture of two different iron oxide phases, hematite $\left(\alpha-\mathrm{Fe}_{2} \mathrm{O}_{3}\right)$ and maghemite $\left(\gamma-\mathrm{Fe}_{2} \mathrm{O}_{3}\right)$, and characterize their magnetic field-driven self-assembly. We demonstrate that the relative amount of the two phases can be adjusted in a continuous way by varying the reaction time during the synthesis, leading to strongly varying magnetic properties of the particles. Not only does the saturation magnetization increase dramatically as the composition of the spindles changes from hematite to maghemite, but also the direction of the induced magnetic moment changes from being parallel to the short axis of the spindle to being perpendicular to it. The magnetic dipolar interaction between the particles can be further tuned by adding a screening silica shell. Small-angle X-ray scattering (SAXS) experiments reveal that at high magnetic field, magnetic dipole-dipole interaction forces the silica coated particles to self-assemble into a distorted hexagonal crystal structure at high maghemite content. However, in the case of uncoated maghemite particles, the crystal structure is not very prominent. We interpret this as a consequence of the strong dipolar interaction between uncoated spindles that then become arrested during field-induced self-assembly into a structure riddled with defects.

Received 22nd June 2017, Accepted 7th September 2017 DOI: $10.1039 / \mathrm{c} 7 \mathrm{nr} 04518 \mathrm{~b}$ rsc.li/nanoscale

\section{Introduction}

The colloidal behaviour of magnetic nanoparticles has attracted the attention of a large number of researchers over the past couple of decades. There has been an extensive effort towards understanding their unique properties, and controlling their behaviour in the presence of a magnetic field. ${ }^{1,2}$ The possibility of directing their self-assembly by an external magnetic field opened up new routes to develop novel stimuliresponsive materials, which have potential applications in many different fields, including photonics, ${ }^{3,4}$ drug delivery, ${ }^{5,6}$

\footnotetext{
${ }^{a}$ Adolphe Merkle Institute and Fribourg Center for Nanomaterials, University of Fribourg, Fribourg, Switzerland

${ }^{b}$ Division of Physical Chemistry, Department of Chemistry, Lund University, Lund, Sweden.E-mail: antara.pal@fkem1.lu.se, peter.schurtenberger@fkem1.lu.se

${ }^{c}$ Institut de Physique de la Matière Condensée, EPF Lausanne, Lausanne, Switzerland ${ }^{d}$ Department of Geosciences, University of Fribourg, Fribourg, Switzerland

${ }^{e}$ Institut fur Geophysik, ETH Zurich, Zurich, Switzerland

$\dagger$ Electronic supplementary information (ESI) available. See DOI: 10.1039/ C7NR04518B

$\$$ These authors contributed equally to this work.

$\S$ Present address: BASF SE, Advanced Materials, Formulation Platform, 67056 Ludwisghafen am Rhein, Germany.
}

pattering, ${ }^{7-11}$ magnetic levitation, ${ }^{12-15}$ imaging, ${ }^{16}$ catalysis, ${ }^{17}$ medicine ${ }^{18-20}$ and DNA separation. ${ }^{21,22}$ This magnetic fieldinduced bottom-up approach is extremely versatile, since it exploits the fast, anisotropic and reversible nature of the magnetic dipole-dipole interaction, which can either be attractive or repulsive in nature depending on the angle between the magnetic field and the line connecting the dipoles.

The magnetic interactions between particles can also be tuned through the addition of a silica layer, which can act as an additional handle to regulate the self-assembly process. Core-shell nanoparticles with a silica coating are of special interest since one can easily modify the silica surface with a large variety of functional groups using silane chemistry to broaden the choice of solvents as well as their potential applications. ${ }^{23-28}$

Although the literature is replete with reports on the fieldresponsive behaviour of spherical magnetic particles, ${ }^{29-33}$ selfassembly of anisotropic hybrid nanoparticles is a comparatively lesser explored avenue till date. ${ }^{2,34,35}$ Ding and co-workers have studied the field driven self-assembly of magnetic ellipsoids into photonic crystals. ${ }^{36,37}$ Their approach was limited to ellipsoids with aspect ratios close to that of a sphere which explains the formation of a distorted FCC crystal and hence the photonic behaviour. Another report involving the magnetic 
field induced self-assembly of anisotropic ellipsoids shows no crystalline but polarised fluid and nematic phases. ${ }^{38}$

Here we present an investigation on the influence of an external magnetic field on the self-assembly behaviour of hybrid magnetic nanoparticles with ellipsoidal shape and tuneable aspect ratio and magnetic properties. These hybrid nanoparticles are composed of two different iron oxide phases, hematite $\left(\alpha-\mathrm{Fe}_{2} \mathrm{O}_{3}\right)$ and maghemite $\left(\gamma-\mathrm{Fe}_{2} \mathrm{O}_{3}\right)$, and exhibit a complex response towards the externally applied field. We follow a synthesis route which allows us to control the relative amount of hematite and maghemite within the particles in a continuous manner. ${ }^{39,40}$ We explore the use of an additional silica layer in order to further tune magnetic interactions and field-driven self-assembly. We show that in the case of coated particles, at high maghemite content, strong dipolar interactions lead to the formation of a distorted hexagonal crystalline structure, while at high hematite content no such selfassembled structure is observed. Interestingly, well-defined structures are prominent only for core-shell particles with an optimal shell thickness. In the absence of the silica shell, the dipolar interaction is strong enough to trap the system in a kinetically arrested state, hindering the formation of an equilibrium crystal structure while for a thicker shell the dipolar interaction is completely screened out to hinder the self assembly process.

\section{Synthesis and characterization methods}

Spindle-shaped hematite ( $\mathrm{SH}$ ) nanoparticles were synthesized using a previously developed method. ${ }^{41}$ Part of these particles were coated with a layer of silica using a method described in ref. 42 and 43. Details about the synthesis can be found in ESI. $\dagger$ Hybrid particles with two different iron oxide phases (i.e., consisting of both hematite and maghemite) were prepared using the procedure as described below. Initially hematite ellipsoids were heated for different time spans, starting from 10 to 240 minutes, under a constant flow of hydrogen in a cylindrical oven maintained at $360^{\circ} \mathrm{C}$ to reduce the hematite into magnetite $\left(\mathrm{Fe}_{3} \mathrm{O}_{4}\right)$. The temperature of the oven was subsequently reduced to $240{ }^{\circ} \mathrm{C}$ and the sample was kept at this temperature for another two hours under a constant flow of air to oxidize magnetite to maghemite. In order to differentiate between the different types of nanoparticles used in our experiments we have adopted the following nomenclature to designate them. In the case of uncoated hematite spindles the prefix $\mathrm{SH}$ is used, while for silica coated hematite we use the prefix SCH. The time of transformation for each particle set is mentioned after the prefix. Thus with this nomenclature, samples bearing the name SH60 refer to particles that were hydrogenated at $360{ }^{\circ} \mathrm{C}$ for 60 minutes and subsequently oxidized at $240{ }^{\circ} \mathrm{C}$ for two hours.

The size and shape characterization of the ellipsoidal colloids was carried out using transmission electron microscopy (TEM) (TEM-CM100 microscope from Philips operating at $100 \mathrm{keV})$.
Particle size distributions were calculated by measuring at least 100 particles from TEM images using Imagetool. High Resolution Transmission Electron Microscopy (HRTEM) (Philips CM200 operating at $200 \mathrm{keV}$ ) was used to investigate the internal structure of particles before and after the hydrogenation. Selected area diffraction patterns were indexed using the JEMS software. Powder X-ray diffraction patterns of the different samples were measured with a (Philips, PW1800) diffractometer (Cu-tube, $40 \mathrm{kV}, 40 \mathrm{~mA}$, step scan mode $0.02^{\circ}$ per step, $2 \mathrm{~s}$ per step, $2 \theta=10^{\circ}-140^{\circ}$ ). Rietveld refinement of the patterns was done with the TOPAS3 software package (Bruker Inc.), using the fundamental parameter approach. Saturation magnetization was defined from hysteresis loops (MH curves) that were measured on a Princeton Measurements Corporation vibrating sample magnetometer (Model 3900).

Small-angle X-ray scattering (SAXS) experiments were carried out at the cSAXS beamline of the Swiss Light Source (Paul Scherrer Institute, Switzerland) with a sample-detector distance of $7 \mathrm{~m}$ and an energy of $8.7 \mathrm{keV}$ (corresponding to a wavelength of bda $=0.1425 \mathrm{~nm}$ ). The scattering patterns were recorded on a single-photon counting PILATUS detector (1475 $\times 1679$ pixels). The samples with a $0.5 \mathrm{wt} \%$ suspension of colloidal particles were sealed in quartz capillaries of $1 \mathrm{~mm}$ diameter and $0.1 \mathrm{~mm}$ wall thickness, and investigated at room temperature. A horizontal magnetic field was produced with a water-thermostated electromagnet such that the field lines were always normal to the incident beam.

\section{Results and discussion}

Fig. 1(a) and (b) show the TEM image of the core-shell particles and the length and width distribution of their cores $(l=$ $308 \pm 38 \mathrm{~nm}, w=55 \pm 7 \mathrm{~nm}$ and aspect ratio of $\rho=5.6)$. The TEM image and the size distribution of the uncoated particles have been included in the ESI (Fig. S1 $\dagger$ ). We have used two different silica shell thickness, namely $20 \mathrm{~nm}$ and $40 \mathrm{~nm}$. TEM measurements also confirmed that the consecutive hydrogenation and oxidation process involving the transformation from hematite to maghemite did not change the overall particle size (data not shown). The Selected Area Electron Diffraction (SAED) patterns and the HRTEM images of the sample SH60 clearly reveals the co-existence of two types of crystal structures (Fig. 1(c) and (d)) in these particles. The lattice fringes at each end of these particles are compatible with the hematite phase, while the ones observed at the central portion of the particle can be attributed to the maghemite phase. The corresponding SAED pattern contains diffraction spots from both hematite (indices in brown) as well as from maghemite (indices in white) (Fig. 1(d)).

In order to quantify the relative amounts of the two different iron oxide species as a function of hydrogenation time, we have carried out X-ray powder diffraction measurements (XRD) after different reaction times (see Fig. S2 in ESI $\dagger$ ). Rietveld refinements of the XRD patterns allow us to obtain the quantitative evolution of both iron oxide phases as the 

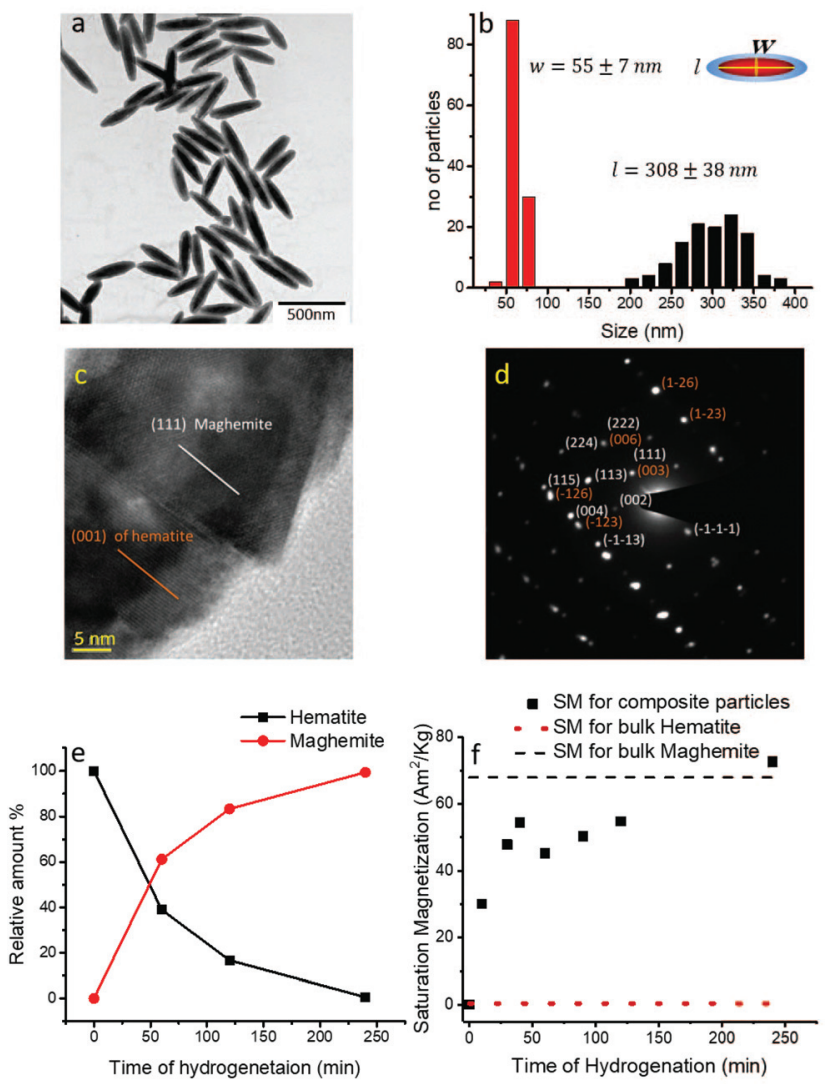

Fig. 1 (a) TEM image of silica coated hematite spindle and (b) the size distribution of their core for thinner coating. (c) HRTEM image of SH60 showing the (100) fringes of hematite (brown line) and the (111) fringes of maghemite (white line). (d) A hybrid spindle can be indexed for hematite (indices in brown) and maghemite phase (indices in white). (e) Variation of hematite and maghemite phase with the hydrogenation time as obtained from the X-ray diffraction measurements. (f) Variation of saturation magnetization with the hydrogenation time as obtained from the MH loop.

transformation progresses as shown in Fig. 1(e). It is clear from Fig. 1(e) that with increasing hydrogenation time, more and more hematite changes to maghemite. HRTEM images, SAED patterns and the quantitative analysis of the XRD data clearly reveal that the internal composition of the hybrid iron oxide spindles can be tailored by selectively choosing the time of hydrogenation.

We have further characterized the bulk magnetic properties of these hybrid anisotropic particles with the help of magnetization (MH-loops) measurements (see Fig. S3 in ESI†). We found that as the conversion time increases, the saturation magnetization $(\mathrm{SM})$ of these hybrid particles steeply increases from a lower value of around $\mathrm{SM} \approx 0.12 \mathrm{Am}^{2} \mathrm{~kg}^{-1}$ for pure hematite particles ${ }^{42,44}$ until it levels off at a value $S M \approx 72$ $\mathrm{Am}^{2} \mathrm{~kg}^{-1}$, which corresponds to the expected value for pure maghemite (Fig. 1(f)). ${ }^{45}$ These results demonstrate that it is possible to tune the SM of the hybrid particles over a large range of values by varying the relative amount of the two consisting species. In the following section, we will discuss their behaviour in the presence of an external magnetic field.
The field-induced alignment and the self-assembly of the partially transformed particles was first studied directly using TEM. Dispersions $(0.5 \mathrm{wt} \%)$ of each type of particles were dried on a TEM grid in the presence of a homogeneous magnetic field $(B \sim 1 \mathrm{~T})$ (Fig. 2). The homogeneity of the field was ensured by using a pair of permanent magnets and by placing the samples at a position equidistant from them. In the case of SH00, i.e., pure hematite spindles, we find that the majority of the particles are oriented with their short axis parallel to the direction of the applied magnetic field, Fig. 2(a). This is a result of the magnetic properties of hematite, where the magnetization is not governed by the particle shape, but by the magnetocrystalline anisotropy. For hematite particles, the magnetization lies in the basal plane, normal to the trigonal axis, and aligns along one of the $a$-axes closest to the applied field. It is very difficult to pull the magnetization out of this basal plane since the susceptibility is 100 times higher in the basal plane compared to that along the $c$-axis. For the spindles, the hematite $c$-axis lies along the long axis of the spindle, which means that the magnetization lies along the short axis

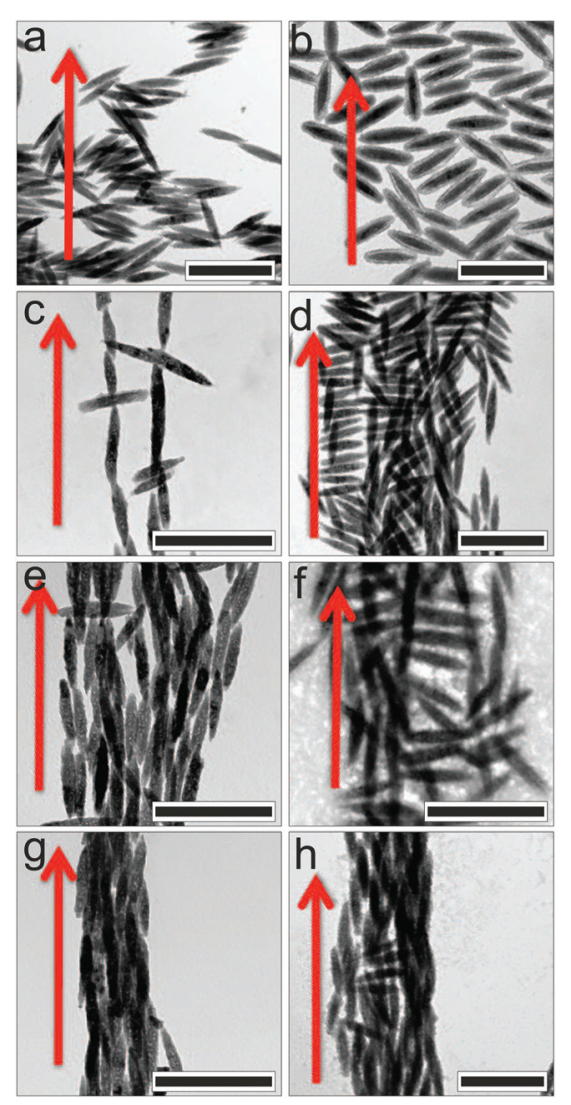

Fig. 2 TEM images for Spindle Hematite (SH) samples with transformation time (a) $0 \mathrm{~min}, \mathrm{SHOO}$ (b) $60 \mathrm{~min}, \mathrm{SH} 60$ (c) $120 \mathrm{~min}, \mathrm{SH} 120$ (d) $240 \mathrm{~min}, \mathrm{SH} 240$ and Silica Coated Hematite $(\mathrm{SCH})$ samples for thinner coating with transformation time (e) $0 \mathrm{~min}, \mathrm{SCH} 00$ (f) $60 \mathrm{~min}, \mathrm{SCH} 60$ (g) $120 \mathrm{~min}, \mathrm{SCH} 120$ and (h) $240 \mathrm{~min}, \mathrm{SCH} 240$ which have been dried on TEM grids in presence of magnetic field. The arrow in red indicates the direction of the external field. The scale bars in the images correspond to $500 \mathrm{~nm}$ 
of the spindle. This alignment leads to a weak magnetic moment parallel to the short particle axis. ${ }^{38,42,44}$ However, the low magnetic moment of hematite and possible drying effects restrict complete alignment of all the particles.

In contrast, for the SH240 particles we find that all of them align parallel to the applied field (Fig. 2(d)). Parallel alignment of the particles along the direction of the magnetic field indicates that the magnetic moment is now along the long particle axis. This, in turn, reflects the almost complete conversion of hematite to maghemite (Fig. 1(e)). The direction of the magnetic moment for particles made up of maghemite, which has a cubic structure, can also be controlled by its atomic crystalline structure, but only if the grain is isomorphic (or equant). In this case the magnetization of each grain wants to lie along the [111] direction of the atomic crystal. As soon as the shape of the grain deviates from isomorphic (deviation $\sim 8 \%$ ), the particle shape starts to control the preferred direction of magnetization because of the high spontaneous magnetization.

In the intermediate steps, SH60 (Fig. 2(b)) and SH120 (Fig. 2(c)), very interesting and more complex assembly patterns are shown. The particle alignment in these cases arises as a consequence of the competition between the magnetic moments of the hematite and maghemite phases within the particles. The hematite phase favors perpendicular alignment. However, as discussed previously, for anisotropic maghemite particles their shape controls the preferred direction of the magnetization. At the beginning of the reduction process, maghemite starts to form in the shape of thin lamellae. Such a thin layer of a maghemite domain with dimensions given by the particle width $w$ and a layer thickness $l_{\text {maghemite }}<w$ then results in an additional magnetic moment along the long axis of the strip which is equivalent to the short axis of the particle. This magnetic moment thus lies parallel to the hematite magnetic moment and enhances a perpendicular alignment, and the resulting stacked self-assembly structure is due to the much larger induced magnetic moment of maghemite. As the hematite to maghemite conversion continues, the lamellae get thicker and the maghemite domain now has a structure where the extension along the long particle axis is larger than along the short axis of the particle, i.e. $l_{\text {maghemite }}>w$, and its magnetization direction switches to the long axis of the spindle. For particles with a more extensive conversion, the maghemite contribution dominates, which results in an overall particle magnetization that lies parallel to the long particle axis, favoring parallel alignment and the formation of chains and bundles in the presence of the magnetic field (see ESI $\dagger$ for a more detailed discussion). As the converted maghemite phase has a relatively broad volume distribution, we thus expect to find mixtures of particles with varying amounts of these three types $\left(l_{\text {maghemite }}=0, l_{\text {maghemite }}<w\right.$ and $\left.l_{\text {maghemite }}>w\right)$ of composition for intermediate hydrogenation times, which then results in the alignment and assembly patterns shown in Fig. 2(b and c).

For the silica coated particles of thinner coating, we see a dominant smectic like side by side stack formation for SCH60 (Fig. 2(f)), which is reduced for SCH120 (Fig. 2(g)), and com- pletely disappears for SCH240 (Fig. 2(h)), where bundles formed by pure maghemite particles dominate. The additional silica shell not only reduces the magnetic dipole interaction, but also slows down the hydrogenation process. Therefore extended maghemite domains with a magnetic moment along the long particle axis appear later in coated compared to uncoated particles.

Since the field-induced self-assembly seen in Fig. 2 is also influenced by the effect of capillary forces that occur in samples during drying, we have investigated the self-assembly behavior in dispersion in situ with SAXS. The SAXS patterns obtained at magnetic field strengths of $3 \mathrm{mT}$ and $1048 \mathrm{mT}$ are displayed in Fig. 3. The left panel in Fig. 3 shows the scattering patterns obtained for SH series while the right panel displays the data for SCH series. The top portion of the right panel represents the data for a thin coated sample, where the silica coating is $20 \mathrm{~nm}$ thick while the bottom panel corresponds to a thicker coating of $40 \mathrm{~nm}$. The scattering patterns are elongated either parallel or perpendicular to the applied field direction as a function of conversion time. This feature is perfectly understandable if one takes into account the fact that the observed SAXS pattern is the representation of the particle alignment in Fourier space. Thus alignment of the spindles with their long axes perpendicular to the applied field in real space manifests as an elongation in the scattering intensity along the direction of the field in Fourier space, whereas the elongation of the pattern in a direction perpendicular to the field in Fourier space implies the alignment of the spindles with their long axis along the field direction in real space.

For pure hematite spindles SH00, the magnetic moment is along the short axes of the spindles, as a result they always orient themselves with their short axis parallel to the applied magnetic field, provided the field is strong enough to interact with the weak magnetic moment of hematite particles as is evident from Fig. $3(\mathrm{~b}) .^{38,42,44}$

SH10 exhibits a slightly anisotropic scattering pattern already at a low magnetic field strength of $3 \mathrm{mT}$, Fig. 3(c). The deviation from the isotropic scattering pattern observed for pure hematite (Fig. 3(a)) indicates that there is a much stronger coupling between the magnetic field and the spindles in SH10. This is possible only if the spindles in SH10 have a magnetic moment which is significantly larger than that of pure hematite - a clear sign for the presence of maghemite domains after 10 minutes of hydrogenation. Another interesting thing to be noted here is the nature of the anisotropy. From Fig. 3(c) one can see that the scattering pattern is not only stretched perpendicular to the magnetic field, but also along the field. This can be understood based on the previous discussion of the TEM pictures shown in Fig. 3. We thus expect that some particles will have only thin strips of maghemite, which extend along the width of the ellipsoid satisfying $l_{\text {maghemite }}<w$. Maghemite being strongly magnetic in nature, these strips contribute a sufficiently large magnetic moment to the overall magnetic moment of the particle to at least partially align them perpendicular to the field, thus resulting in a stretching of the diffraction pattern along the field. The 

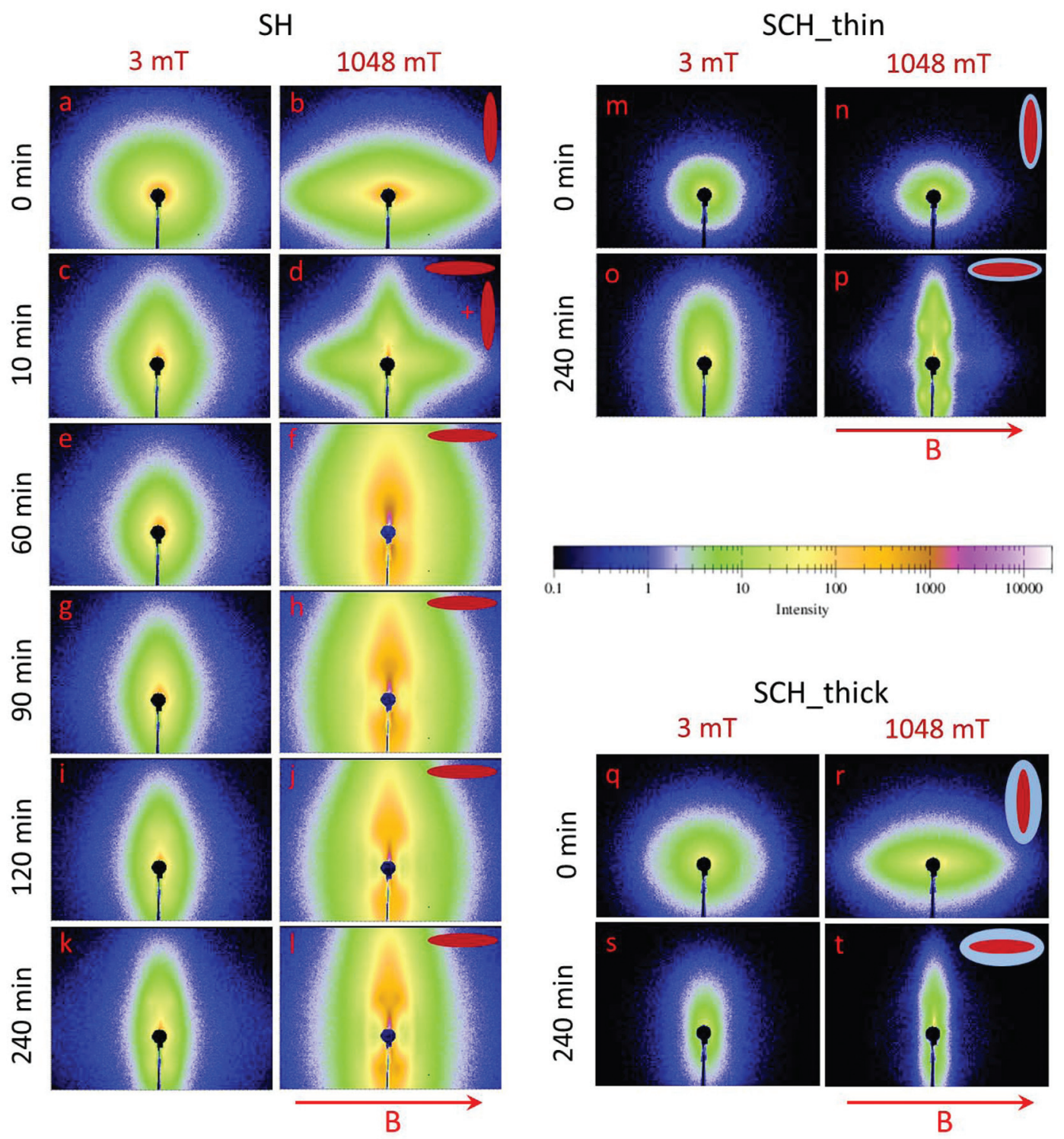

Fig. 3 SAXS diffraction patterns measured for uncoated (left) and coated (right) iron oxide spindles for different field strengths and transformation times as mentioned in the figure. The top portion of the right panel corresponds to a thinner silica coating of $20 \mathrm{~nm}$ while the bottom part represents a thicker coating of $40 \mathrm{~nm}$. The applied magnetic field is along the horizontal direction.

stretching of the diffraction pattern perpendicular to the magnetic field can be understood by the presence of spindles with maghemite domains which satisfies $l_{\text {maghemite }}>w$ (see ESI $\dagger$ for a more detailed discussion). This feature is even more clear for higher magnetic field (Fig. 3(d)) when one can easily observe the stretching of the diffraction pattern in both the directions, confirming the presence of two different types of compositions satisfying $l_{\text {maghemite }}<w$ and $l_{\text {maghemite }}>w$. At higher conversion times (60 $\mathrm{min}$ and $90 \mathrm{~min}$ ) and at higher field strengths, all particles are almost completely aligned, which means that those with a magnetic moment perpendicular to the long axis are still able to freely rotate around the short axis, while those with the magnetic moment parallel to the long axis will all have a unique orientation with the long axis parallel to the field direction. The scattering contribution from the particles where the maghemite domains satisfy $l_{\text {maghemite }}>w$ thus dominates, and the scattering pattern displays a strong anisotropy perpendicular to the field (Fig. 3(f) and (h)). ${ }^{44}$

In the case of SH120, the amount of the hematite phase has decreased significantly, and one observes a strongly anisotropic pattern in the direction perpendicular to the magnetic field at low as well as at high magnetic field strengths (Fig. 3(i and j)), in agreement with our previous quantitative measurement of the relative amount of the two iron oxides present in the spindles (Fig. 1(e)). For SH240, an even stronger alignment of the magnetic nanoparticles in the direction of the field can be observed from the scattering intensity (Fig. 3(k and l)).

Moreover, a qualitative comparison between the scattering patterns at high field strength already clearly indicates that the presence of the field not only leads to an orientation of the 
particles, but also causes considerable field-driven self-assembly into larger structures, such as chains and bundles that result in a very strong forward scattering and a much more complex anisotropic scattering than the ones obtained from the form factor of individual aligned ellipsoids.

We have also investigated the field-induced orientation and self-assembly of silica coated composite hematite-maghemite particles. For this purpose we have used two different thickness of silica coating, namely $20 \mathrm{~nm}$ and $40 \mathrm{~nm}$. An isotropic scattering pattern is observed for both types of coated pure hematite particles at $3 \mathrm{mT}$, which indicates a random orientation of the anisotropic nanoparticles (Fig. 3(m and q)). This is in good agreement with the expectation for hematite particles with a weak magnetization at low field strengths (3 mT). At high external field strengths, however, we observe a weak orientation of the short axis of the particles along the applied field direction, similar to the previous measurements with uncoated hematite particles (Fig. 3(n and r)).

The situation changes quite dramatically for the partially transformed SCH particles. At high magnetic field, for a hydrogenation time of $240 \mathrm{~min}$, the diffraction patterns get highly elongated perpendicular to the magnetic field for both thinner and thicker coating (Fig. 4(p and $\mathrm{t}$ )). In addition, for the thinner coating, new features in the form of diffraction spots have developed, indicating the formation of a crystal structure.
It is worth noting that the higher order reflections are not present in this case. This can be attributed to the size polydispersity in the nanoparticles as well as the fact, that the bundle structure and the form factor of the individual particles leads to a strong decrease of the intensity at higher values of $q$. However, for the thicker coating, no such crystal formation is observed. We believe that in this case, the silica coating is thick enough to screen out the dipolar interaction completely to hinder the formation of crystals.

The field-induced assembly into regular structures is further illustrated in Fig. 4(a and b), which show the diffraction patterns along with the $d$-spacings for the structures formed by thinner coated $\mathrm{SCH} 240$ and $\mathrm{SH} 240$ at maximum field strength (1048 $\mathrm{mT})$ together with two models proposed to interpret the experimental data (Fig. 4(c and d)). In the presence of the magnetic field, silica-coated hematite-maghemite particles form a crystalline structure which can be modeled as a distorted hexagonalone (Fig. 4(c)). Lattice spacings obtained from the diffraction pattern match well with the expected values for the particle size. Due to the dipolar repulsion between the consecutive particles in two rows, the spacing along the field should be less than the length of the particles. However we did not observe any diffraction peak along the field direction. This can be attributed to the presence of a form factor minimum, which when superimposed with the
C.

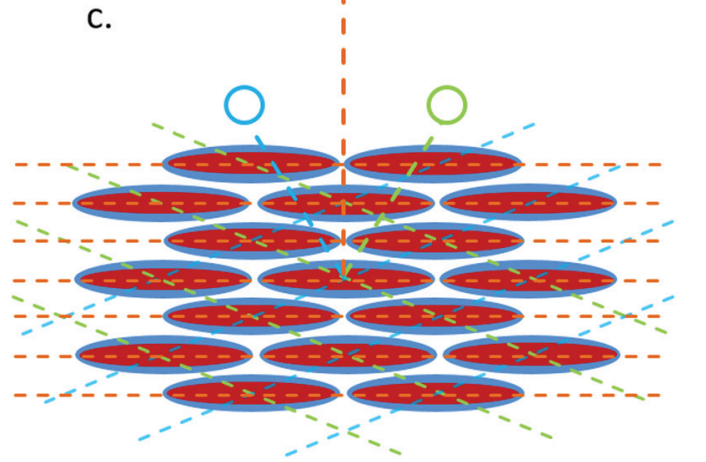

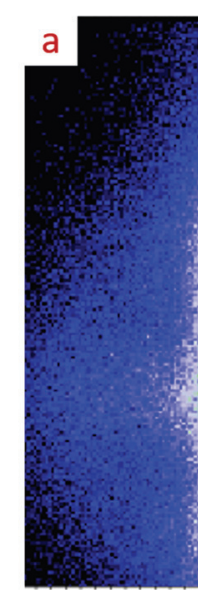

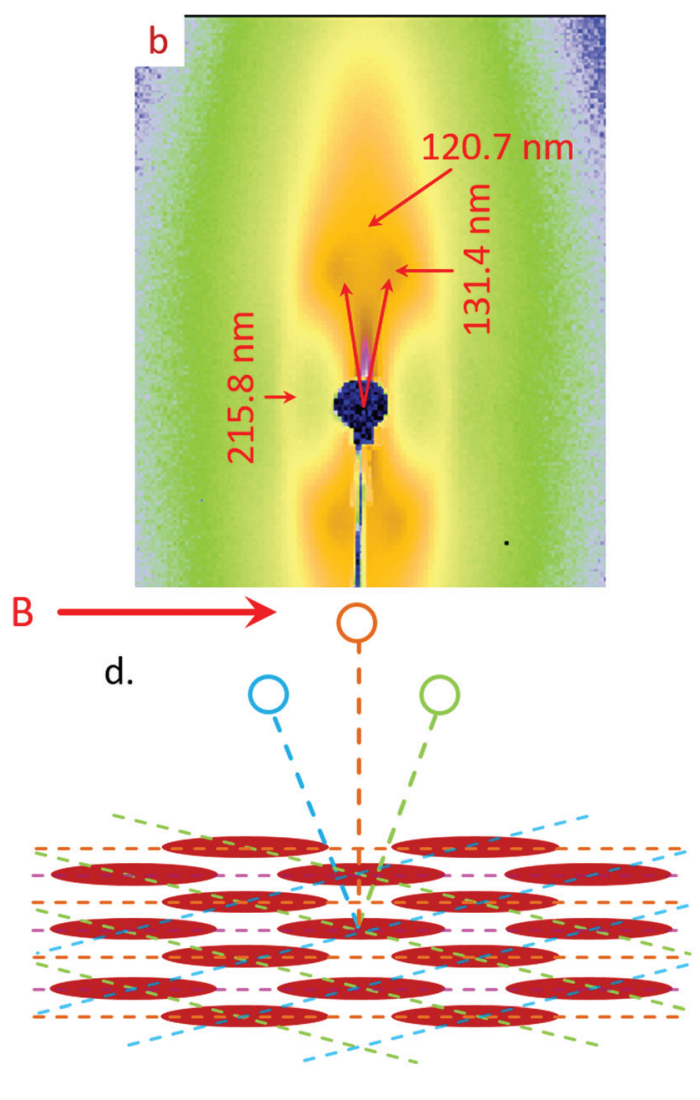

Fig. 4 SAXS patterns for Silica Coated Hematite samples (a) SCH240 at $1048 \mathrm{mT}$ and (b) SH240 at $1048 \mathrm{mT}$ along with the different $d$-spacings. Schematic showing the magnetic field mediated assembly in real space for (c) $\mathrm{SCH} 240$ and (d) $\mathrm{SH} 240$. 
structure factor peak leads to a considerable reduction in intensity so as to render it invisible. In the perpendicular direction the spacing should be the width of the particles, which matches with the obtained value. The other two peaks arise from the distorted hexagonal-like packing. Based on these experimentally obtained peak positions we propose a model which is shown in Fig. 4(c).

For uncoated particles, we only observe some broad diffraction peaks, which indicate the formation of a similar lattice that is however riddled with defects (Fig. 4(b)). It is not possible to ascertain the peak positions as well as the $d$-spacings very precisely. We speculate that the much stronger dipolar interactions between the uncoated particles results in dynamical arrest during the self-assembly process and the formation of non-equilibrium structures full of defects. However, for uncoated particles the diffraction peak along the field direction is very clear, which indicates that all the particles are aligned along this direction. ${ }^{46}$ The peak corresponds to a repeat distance of $215.8 \mathrm{~nm}$, which is significantly larger than the value expected from the length of the particles along this direction for the coated ones. This is because of the fact that the silica coating partly screens out the dipolar interactions, allowing the particles to form a more compact structures in the presence of the coating. For coated particles the two nearest neighbors which belong to two consecutive lines of particles can come much closer than for the uncoated ones (Fig. 4(c and d)). This is further supported by the increase in angle between the green and blue peaks for the coated particles, which also indicates the formation of a more compact assembly when the particles are subjected to a silica coating.

It is worth discussing in more detail the consequences of the fact that for anisotropic particles the conversion from hematite to maghemite influences the particles and their resulting superstructure in two ways. Increasing the conversion not only enhances the net magnetization, but as discussed above also the direction of the induced magnetic moment with respect to the particle frame. As the system will respond to the application of an external field in two ways - alignment of the anisotropic particles due to particle-field and formation of self assembled structure due to the effective particle-particle interactions - this has important consequences for the formation of field-induced superstructures. For pure hematite ellipsoids, dipolar interactions are negligible, the only interactions to consider are excluded volume and (weak) screened Coulomb interactions, and the dominating field effect is then an enhanced alignment of the particles. However, as the magnetic moment is perpendicular to the long particle axis, particles are still able to freely rotate with their long axis around the magnetic moment. While the field constrains the short axes of the ellipsoids, the repulsive particle-to-particle interaction also constrains the long axes of the ellipsoids, and the combined effects of both interactions then result in a situation where positional correlations are coupled to field-induced orientational correlations. As described in, ${ }^{38}$ at intermediate volume fractions this then results in the formation of a rather peculiar nematic-like layered suspension structure, with liquid-like structural correlations along the field axis, but only weak positional order within the alignment plane perpendicular to the field.

When now performing a partial conversion of the hematite particles to maghemite, the formation of thin maghemite lamellae within the hematite ellipsoids results in an additional contribution to the magnetic moment parallel to the one from hematite, and the alignment features thus do not change qualitatively. However, the much larger susceptibility of maghemite results in a larger magnitude of the particle magnetic moment, and thus a larger dipolar contribution to the particle-particle interactions. For the particles used in this study this additional interaction term does not result in the formation of a self-assembled superstructure in suspension at the concentrations investigated, but the results from the TEM experiments where the samples were prepared in the presence of a magnetic field clearly indicates the posibility for forming regular stacked superstructures at higher densities as achieved in the drying step on the microscopy grid (Fig. 2f).

For a more extensive or complete conversion, we finally observe a qualitatively different behaviour. For a given field strength, the particle magnetic moment has not only dramatically increased, but also switched direction, and is now parallel to the long axis. In the presence of sufficiently strong external magnetic fields these particles are then forced to align along the field direction, and the dipolar interactions between particles are strong enough to induce the formation of selfassembled superstructures already at the low concentrations investigated in this study. While interparticle interactions for pure maghemite spindles are too large for the formation of highly ordered crystalline arrays for the used field strength, the addition of a silica shell with variable thickness then allows us to optimise field-directed self assembly and form well ordered supracrystals with sufficient degree of ordering to exhibit welldefined Bragg peaks in a SAXS experiment. While we have not performed an exhaustive study of the self assembly of fully converted maghemite ellipsoids as a function of shell thickness, we would expect a behaviour where the resulting superstructures evolve from the defect-riddled distorted hexagonal to highly ordered crystalline structures to finally a simple fieldinduced nematic phase once the silica shell is thick enough to effectively screen the dipolar interactions between particles, and where we are then left with a simple combination of a field-induced alignment and excluded volume interactions. This clearly indicates the potential of the chosen system for field-driven self assembly, where the resulting structures are formed as a result of the combined and strongly coupled effects of field-induced particle alignment and particle interactions, which can be finely tuned via the internal composition of the hybrid magnetic particles.

\section{Conclusion}

We have discussed a versatile route to synthesize anisotropic hybrid magnetic iron oxide nanoparticles consisting of hema- 
tite and/or maghemite phases in varying proportions. As a result of the significantly different magnetic properties of these two phases, both in terms of the magnitude as well as the direction of the resulting magnetic moments, this provides us with particles whose magnetic properties can be tuned to a large degree. Moreover, adding a silica coating with tunable thickness results in an additional variability in the final particle shape and interaction potential. This provides us with nanoparticle building blocks for a highly versatile driven self assembly into various ordered morphologies. In the case of magnetic field-driven self-assembly, the resulting lattice parameters and quality of the crystalline assembly clearly show that we can use a combination of the hematite-maghemite conversion and an added silica shell to carefully tune particle shape and inter particle interactions for optimal self-assembly. A comparison between the results from TEM and SAXS also has demonstrated that the tunable particle anisotropy, together with the use of external magnetic fields to either control the particle orientation while utilizing capillary interactions in a drying sample or to generate strong magnetic dipolar interactions, allows us create different self-assembled structures. We believe that the insight gained into fielddirected self-assembly from a combination of different methods with the required spatial resolution for these anisotropic hybrid particles has set the stage for future and more systematic investigations of the combined role of the shape, internal composition, particle concentration and field strength. This may lead to materials with interesting mechanical, optical and magnetic properties in particular at higher particle concentrations, where ellipsoidal particles are expected to have a quite rich phase diagram. ${ }^{38}$

\section{Conflicts of interest}

There are no conflicts to declare.

\section{Acknowledgements}

We are grateful to Liliane Ackermann Hirschi for helping us in the synthesis of the hematite nanoparticles and Benoit Droz for the help in carrying out the phase transformation of nanoparticles. The SAXS experiments were conducted at the cSAXS beamline of the Swiss Light Source, Paul Scherrer Institute, Switzerland, and we acknowledge the expert help from the local contact, Dr Andreas Menzel. Financial support from the Adolphe Merkle Foundation, the Swiss National Science Foundation, the European Research Council (ERC-339678COMPASS), and the Knut and Alice Wallenberg Foundation (project grant KAW 2014.0052) is gratefully acknowledged.

\section{References}

1 A.-H. Lu, E. Salabas and F. Schüth, Angew. Chem., Int. Ed., 2007, 46, 1222.
2 M. Grzelczak, J. Vermant, E. M. Furst and L. M. LizMarzan, ACS Nano, 2010, 4, 3591.

3 X. Xu, G. Friedman, K. D. Humfeld, S. A. Majetich and S. A. Asher, Adv. Mater., 2001, 13, 1681.

4 X. Xu, S. A. Majetich and S. A. Asher, J. Am. Chem. Soc., 2002, 124, 13864.

5 M. Chorny, I. Fishbein, B. B. Yellen, I. S. Alferiev, M. Bakay, S. Ganta, R. Adamo, M. Amiji, G. Friedman and R. J. Levy, Proc. Natl. Acad. Sci. U. S. A., 2010, 107, 8346.

6 Z. G. Forbes, B. B. Yellen, K. A. Barbee and G. Friedman, IEEE Trans. Magn., 2003, 39, 3372.

7 K. H. Li and B. B. Yellen, Appl. Phys. Lett., 2010, 97, 083105.

8 C. Ooi, R. M. Erb and B. B. Yellen, J. Appl. Phys., 2008, 103, $07 \mathrm{E} 910$.

9 B. B. Yellen and G. Friedman, J. Appl. Phys., 2003, 93, 8447.

10 B. B. Yellen and G. Friedman, Langmuir, 2004, 20, 2553.

11 B. B. Yellen, O. Hovorka and G. Friedman, Proc. Natl. Acad. Sci. U. S. A., 2005, 102, 8860.

12 K. A. Mirica, S. T. Phillips, C. R. Mace and G. M. Whitesides, J. Agric. Food Chem., 2010, 58, 6565.

13 K. A. Mirica, S. T. Phillips, S. S. Shevkoplyas and G. M. Whitesides, J. Am. Chem. Soc., 2008, 130, 17678.

14 K. A. Mirica, S. S. Shevkoplyas, S. T. Phillips, M. Gupta and G. M. Whitesides, J. Am. Chem. Soc., 2009, 131, 10049.

15 N. D. Shapiro, K. A. Mirica, S. Soh, S. T. Phillips, O. Taran, C. R. Mace, S. S. Shevkoplyas and G. M. Whitesides, J. Am. Chem. Soc., 2012, 134, 5637.

16 J.-H. Lee, Y.-M. Huh, Y.-w. Jun, J.-w. Seo, J.-t. Jang, H.-T. Song, S. Kim, E.-J. Cho, H.-G. Yoon, J.-S. Suh, et al., Nat. Med., 2007, 13, 95.

17 M. Ye, Q. Zhang, Y. Hu, J. Ge, Z. Lu, L. He, Z. Chen and Y. Yin, Chem. - Eur. J., 2010, 16, 6243.

18 J.-H. Lee, K. Lee, S. H. Moon, Y. Lee, T. G. Park and J. Cheon, Angew. Chem., 2009, 121, 4238.

19 C. Xu, K. Xu, H. Gu, X. Zhong, Z. Guo, R. Zheng, X. Zhang and B. Xu, J. Am. Chem. Soc., 2004, 126, 3392.

20 A.-H. Lu, E. e. Salabas and F. Schüth, Angew. Chem., Int. Ed., 2007, 46, 1222.

21 X. Zhao, R. Tapec-Dytioco, K. Wang and W. Tan, Anal. Chem., 2003, 75, 3476.

22 S. V. Sonti and A. Bose, Colloids Surf., B, 1997, 8, 199.

23 J. Ge, Y. Hu and Y. Yin, Angew. Chem., 2007, 119, 7572.

24 J. Ge, H. Lee, L. He, J. Kim, Z. Lu, H. Kim, J. Goebl, S. Kwon and Y. Yin, J. Am. Chem. Soc., 2009, 131, 15687.

25 L. He, Y. Hu, X. Han, Y. Lu, Z. Lu and Y. Yin, Langmuir, 2011, 27, 13444.

$26 \mathrm{~J} . \mathrm{Ge}$ and Y. Yin, Adv. Mater., 2008, 20, 3485.

27 J. Ge, J. Goebl, L. He, Z. Lu and Y. Yin, Adv. Mater., 2009, 21, 4259.

28 M. Wang, L. He, Y. Hu and Y. Yin, J. Mater. Chem. C, 2013, 1, 6151.

29 V. Malik, A. V. Petukhov, L. He, Y. Yin and M. Schmidt, Langmuir, 2012, 28, 14777.

30 A. Pal, V. Malik, L. He, B. H. Erné, Y. Yin, W. K. Kegel and A. V. Petukhov, Angew. Chem., 2015, 127, 1823. 
31 M. Klokkenburg, C. Vonk, E. M. Claesson, J. D. Meeldijk, B. H. Erné and A. P. Philipse, J. Am. Chem. Soc., 2004, 126, 16706.

32 M. Klokkenburg, B. H. Erné, J. D. Meeldijk, A. Wiedenmann, A. V. Petukhov, R. P. Dullens and A. P. Philipse, Phys. Rev. Lett., 2006, 97, 185702.

33 K. Butter, P. Bomans, P. Frederik, G. Vroege and A. Philipse, Nat. Mater., 2003, 2, 88.

34 M. Wang, L. He, S. Zorba and Y. Yin, Nano Lett., 2014, 14, 3966-3971.

35 M. Wang, C. Gao, L. He, Q. Lu, J. Zhang, C. Tang, S. Zorba and Y. Yin, J. Am. Chem. Soc., 2013, 135, 1530215305.

36 T. Ding, K. Song, K. Clays and C.-H. Tung, Adv. Mater., 2009, 21, 1936.

37 T. Ding, K. Song, K. Clays and C.-H. Tung, Langmuir, 2010, 26, 11544.

38 I. Martchenko, J. J. Crassous, A. M. Mihut, E. Bialik, A. M. Hirt, C. Rufier, A. Menzel, H. Dietsch, P. Linse and P. Schurtenberger, Soft Matter, 2016, 12, 8755.
39 H. Chen, D. C. Colvin, B. Qi, T. Moore, J. He, O. T. Mefford, F. Alexis, J. C. Gore and J. N. Anker, J. Mater. Chem., 2012, 22, 12802.

40 J. J. Crassous, A. M. Mihut, H. Dietsch, O. Pravaz, L. Ackermann-Hirschi, A. M. Hirt and P. Schurtenberger, Nanoscale, 2014, 6, 8726.

41 M. Ocaña, M. Morales and C. Serna, J. Colloid Interface Sci., 1999, 212, 317.

42 M. Reufer, H. Dietsch, U. Gasser, B. Grobety, A. Hirt, V. K. Malik and P. Schurtenberger, J. Phys.: Condens. Matter, 2011, 23, 065102.

43 C. Graf, D. L. Vossen, A. Imhof and A. van Blaaderen, Langmuir, 2003, 19, 6693.

44 M. Reufer, H. Dietsch, U. Gasser, A. M. Hirt, A. Menzel and P. Schurtenberger, J. Phys. Chem. B, 2010, 114, 4763.

45 N. C. Feitoza, T. D. GonÃČ Âğalves, J. J. Mesquita, J. S. Menegucci, M.-K. M. Santos, J. A. Chaker, R. B. Cunha, A. M. Medeiros, J. C. Rubim and M. H. Sousa, J. Hazard. Mater., 2014, 264, 153.

46 V. R. Dugyala and M. G. Basavaraj, RSC Adv., 2015, 5, 60079. 